\title{
DEPORTE EN FAMILIA COMO PREVENCIÓN DE PROBLEMAS DE CONDUCTA DESDE EDADES TEMPRANAS
}

\author{
Alicia Benavides Nieto \\ Miriam Romero López \\ María Fernández Cabezas \\ M. Carmen Pichardo Martínez \\ Departamento de Psicología Evolutiva y de la Educación, Universidad de Granada, Granada, España. \\ aliciabn@ugr.es
}

https://doi.org/10.17060/ijodaep.2017.n1.v3.1014

Fecha de Recepción: 28 Febrero 2017

Fecha de Admisión: 1 Abril 2017

\section{RESUMEN}

En los últimos años los problemas de conducta en edad infantil han aumentado, condicionando la probabilidad de que aparezcan situaciones problemáticas que comprometan el ajuste personal y social de los menores, cristalizando en conductas delictivas o acoso. Para responder a esta problemática, investigaciones recientes aportan evidencias científicas acerca de diferentes intervenciones resaltando la influencia de los estilos educativos parentales en la etapa infantil y del papel del deporte en el fomento de conductas prosociales y de autocontrol. Por ello, el objetivo de la presente investigación es, por un lado, conocer la relación existente entre los estilos educativos parentales, evaluados desde la perspectiva infantil, y los problemas de conducta en niños de preescolar y, por otro, reflexionar sobre los beneficios de la práctica de deporte en familia desde una perspectiva democrática, comprensiva y constructiva en la prevención de problemas de conducta en la etapa preescolar. En el estudio participan 141 alumnos/as (81 niñas y 63 niños) de 4 y 5 años de Granada. Los estilos educativos se evalúan a través de la escala PEF-H de Alonso y Román (2003), y los problemas de conducta mediante tres escalas del BASC de Reynolds y Kamphaus (1992), adaptada al español por González, Fernández, Pérez, y Santamaría (2004). Los resultados obtenidos muestran que el estilo democrático correlaciona significativa y negativamente con los problemas de atención. Sin embargo, no se ha encontrado relación significativa entre el estilo permisivo y los problemas de conducta. En cuanto al estilo autoritario, se encuentra una relación positiva significativa con los problemas de atención. En conclusión, el estilo democrático familiar se presenta como factor de protección para el desarrollo óptimo desde edades tempranas, siendo la práctica del deporte en familia un aspecto positivo para fomentar conductas prosociales, respetuosas, emprendedoras, de autocontrol y críticas, como prevención de problemas de conducta. 


\section{DEPORTE EN FAMILIA COMO PREVENCIÓN DE PROBLEMAS DE CONDUCTA DESDE EDADES TEMPRANAS}

Palabras clave: Intervención familiar, Estilos educativos parentales, Problemas de conducta, Psicología del deporte, Edades tempranas, Educación infantil.

\section{ABSTRACT}

\section{Sport in family as prevention of behavior problems from Early Childhood}

In recent years, behavioral problems in early childhood have increased, conditioning the probability that problematic situations appear that compromises the personal and social adjustment of children, crystallizing in criminal behavior or harassment. In order to respond to this problem, recent studies provides scientific evidence about different interventions highlighting the influence of parenting styles in the infantile stage and the role of the sport in the promotion of prosocial conducts and self-control. Therefore, the objective of this article is, on the one hand, to know the relationship between the parenting styles, assessed form a child perspective, and preschoolers behavior problems and, on the other hand, to reflect on the benefits of family sport practice from a democratic, comprehensive and constructive perspective in preventing behavior problems in the preschool stage. The study involved 141 students ( 81 girls and 63 boys) aged 4 and 5 years old from Granada. Parenting styles are evaluated through PEF-H scale from Alonso y Román (2003), and behavioral problems through three BASC scales from Reynolds y Kamphaus (1992), adapted to spanish by González, Fernández, Pérez, y Santamaría (2004). The results show that the democratic style correlates significantly and negatively with attention problems. However, no significant relationship has been found between permissive style and behavioral problems. As for the authoritarian style, there is a positive and significant relationship with behavior problems. In conclusion, family democratic style is a protective factor for optimal development from an early age, being family sport practice a positive aspect to promote prosocial, respectful, entrepreneurial, self-control and critical conducts, as prevention of behavioral problems.

Keywords: Family Intervention, Parenting styles, Behavior problems, Sport Psychology, Early childhood, Early childhood education.

Los problemas de conducta se pueden definir como comportamientos que se desvían de la norma interfiriendo o bloqueando el desarrollo personal del niño y de su entorno (Cooper, 2011). Los problemas de conducta en edades tempranas representan en la actualidad una grave preocupación para la sociedad. Tanto familias como docentes advierten este aumento de frecuencia en los últimos años. El National Institute for Health and Clinical Excelence (NICE, 2014) identifica como los comportamiento más comunes en las primeras edades los trastornos de la conducta y el comportamiento antisocial. Asimismo, Dery, Toupin, Pauze, y Verlaan (2004) afirman que más de la mitad de las consultas clínicas son por problemas o trastornos de comportamiento. Los problemas conductuales pueden dividirse entorno a dos áreas según Achenbach y Edelbrock (1978): por un lado la internalizante, que incluye somatizaciones como la ansiedad, la depresión, el retraimiento y las preocupaciones somáticas; y por otro lado, el área externalizante, que alude a la falta de control y a la aparición de sintomatología agresiva e hiperactiva. Durante la etapa infantil los síntomas psicopatológicos más frecuentes con relación a los problemas internalizantes son la ansiedad y la depresión asociados a la conducta evitativa (Kendall, Hedtke, y Aschenbrand, 2006). Por su parte, los problemas externalizantes más comunes en preescolares son la desobediencia, la agresión hacia los pares, un alto nivel de actividad incontrolada y una pobre regulación de impulsos (Campbell, 1995).

Estos problemas de conducta desde edades tempranas dificultan la adaptación social de los niños en su entorno (Romero, Robles, y Lorenzo, 2006), pudiendo progresar hacia conductas violentas, de acoso o delincuencia si no se interviene a tiempo (Farrington, 2015). En este marco de 
actuación-intervención situamos a la familia como eje central de influencia. Entendiendo los factores de riesgo como aquellos que condicionan de forma psicológica y/o social la probabilidad de que aparezca una determinada conducta, situación o problema que comprometa el ajuste personal y social de los hijos, y como factores de protección aquellos que intervienen en la respuesta de los hijos ante algún riesgo que predispone un resultado adaptativo (Rodrigo, Máiquez, Martín, y Byrne, 2008; Rutter, 1985); esta influencia familiar puede actuar respectivamente en detrimento del óptimo desarrollo infantil 0, por el contrario, ayudar a desarrollar un mejor ajuste personal y social.

Desde el modelo ecológico transacional de Cicchetti y Lynch (1993), apoyado en el modelo ecológico de Bronfenbrenner (1987), se describen una serie de factores de riesgo y de protección que interactúan dinámicamente dentro de cada sistema (microsistema, mesosistema, exosistema y macrosistema) e influyen en los niveles circundantes. Entendiendo a la familia desde un enfoque ecológico transacional se afirma que hay factores de riesgo y de protección en la unidad familiar que interactúan, de forma transitoria o permanente, dentro de cada sistema e influyen sobre los otros (Rodrigo et. al, 2008).

Asimismo, según el modelo de aprendizaje social de Bandura (1989) los niños en la primera infancia perciben a sus padres como modelos sin replantearse moralmente su actuación o sin comparar con otros modelos familiares diferentes. Farrington (2015) establece la crianza de los hijos 0 los estilos educativos parentales como un factor influyente en la delincuencia.

Tras esta breve revisión podemos afirmar el papel esencial que tiene la unidad familiar en el desarrollo óptimo de los niños en la etapa infantil. En cuanto a su relación con los problemas de conducta, varias investigaciones (Carrasco, y González, 2006; Flouri, y Midouhas, 2017; Raya, Pino, y Herruzo, 2009; Tur, Mestre, y Del Barrio, 2004) ponen de manifiesto una relación significativa con los estilos educativos parentales, demostrando que un estilo educativo excesivamente permisivo 0 autoritario favorece la aparición de conductas agresivas en el niño, mientras que un estilo autoritativo o democrático (altos niveles de apoyo, supervisión y flexibilidad) interviene como factor protector de la conducta agresiva. Así pues, un estudio longitudinal llevado a cabo recientemente por Flouri, y Midouhas (2017) en Reino Unido con una muestra de 16.916 niños evaluados a los 3, 5 y 7 años presenta evidencias significativas acerca de la relación entre una disciplina severa y los problemas de conducta a lo largo del tiempo. Estos autores afirman que una disciplina severa predice problemas emocionales y de conducta en niños de familias tanto de alto como de bajo riesgo social, moderando el efecto de la pobreza y las dificultades.

Descrita la dinámica relación entre los estilos educativos parentales y los problemas de conducta en edad infantil, siguiendo a Dishion y Patterson (1992), se garantiza la eficacia y el beneficio de una intervención temprana, como estrategia preventiva, frente a estrategias correctivas posteriores cuando ya se han presentado problemas de comportamiento. Por su parte, desde la psicología del deporte, numerosas evidencias científicas (Gutiérrez, 2004; Holt, 2016; Miller, 2015; Pelegrín, Garcés y Cantón, 2010) nos muestran los beneficios de la práctica del deporte en el desarrollo de habilidades prosociales y de autorregulación así como la forma de desarrollar una óptima autoestima. En un estudio realizado por Pelegrín, Garcés y Cantón (2010) en el que participaron 507 escolares, comparando entre un grupo que practica deporte frente a otro que no, se observan más conductas prosociales, extrovertidas, respetuosas, disciplinadas, de autocontrol, de liderazgo y autoconfianza, y menos conductas antisociales y delictivas en el grupo que practica deporte frente al que no.

Se establece, por tanto, el deporte en familia como factor de protección para prevenir problemas conductuales infantiles. Es importante tener en cuenta que no es válido cualquier tipo de deporte en familia, sino aquel en el que se ponga en práctica un estilo parental democrático, en el que se 
incentive la toma de decisiones, el respeto de las las reglas acordadas, a fomentar el juego en equipo y a resolver de manera adecuada los conflictos. En cambio, la metodología tradicional en la enseñanza de los deportes usa principalmente la instrucción directa, observándose una escasa implicación en el desarrollo cognitivo y motriz de quien practica el deporte, poniendo énfasis en la adquisición de habilidades técnicas individuales (Galatti, Reverdito, Scaglia, Pae, y Seoane, 2014), promocionando una pobre toma de decisiones (Valera, Ureña, Ruíz, y Alarcón, 2010). Sin embargo, las metodologías centradas en el juego, comprensivas y constructivistas promueven el uso de estilos de enseñanzas que implican cognitivamente a quien realiza el deporte mediante el empleo del descubrimiento y la resolución de problemas (Abad, Benito, Giménez, y Robles, 2013), persiguiendo una mayor implicación y participación y mejorando la toma de decisiones tácticas (Balakrishnan, Rengasamy, y Aman, 2011).

En definitiva, la práctica del deporte desde una metodología comprensiva-constructivista (Alarcón, Cárdenas, Miranda, Ureña y Piñar, 2010), más relacionada con un estilo educativo democrático 0 equilibrado es la que se persigue en el modelo de prevención de problemas de conducta.

\section{OBJETIVOS}

Tal y como se ha argumentado previamente, parece evidente que el estilo educativo democrático 0 equilibrado es el que previene los problemas de conducta desde edades tempranas, por lo que este estudio se centra en conocer esta relación desde la perspectiva infantil y reflexionar acerca de los beneficios del deporte en familia como estrategia preventiva. Por tanto, el objetivo principal se operativiza en torno a dos vertientes: por un lado, conocer la relación entre los estilos educativos parentales evaluados desde la perspectiva infantil y los problemas de conducta en niños y niñas de 4 y 5 años y, por otro lado, reflexionar sobre los beneficios de la práctica de deporte en familia desde una perspectiva democrática, comprensiva y constructiva en la prevención de problemas de conducta en la etapa preescolar.

\section{PARTICIPANTES}

En la presente investigación han participado 141 alumnos/as (63 niños y 81 niñas) de 4 y 5 años de edad, procedentes de dos centros educativos, de similares características, situados en un entorno socioeconómico medio de la provincia de Granada (España).

\section{METODOLOGÍA E INSTRUMENTOS UTILIZADOS}

Teniendo como premisa los objetivos de investigación, la metodología empleada es de tipo ex pos facto prospectivo.

Se ha realizado una correlación de Pearson para analizar la relación entre las distintas variables de los problemas de conducta y los estilos educativos evaluados desde la perspectiva infantil.

Los diferentes análisis se han llevado a cabo a través del programa estadístico Statistical Package for the Social Sciences (SPSS) en su versión 20.0 para Windows.

Para la evaluación de las diferentes variables, se han utilizado los siguientes instrumentos:

Los Problemas de conducta en Educación Infantil se han evaluado a través de la escala BASC, Sistema de Evaluación de la Conducta de Niños y Adolescentes creada por Reynolds y Kamphaus (1992), adaptada al español por González, Fernández, Pérez, y Santamaría (2004). Está constituida por una escala tipo Likert, con cuatro posibilidades de respuesta, desde 0, "nunca", hasta 3 , "casi siempre". Se completa por los tutores o los padres, previa observación del comportamiento de los niños y está compuesta por 14 escalas clínicas y 5 dimensiones globales. Para la presente investigación sólo se utilizarán 37 ítems, correspondientes a las siguientes escalas clínicas: Agresividad, consta de 13 ítems y evalúa la tendencia de actuar de forma hostil (sea verbal o físicamente) que 
amenaza a otros; Hiperactividad, consta de 16 ítems y evalúa la tendencia a ser excesivamente activo, a ejecutar precipitadamente los trabajos y actividades y a actuar sin pensar; y Problemas de atención, consta de 8 ítems y evalúa la tendencia a distraerse fácilmente y la incapacidad para concentrarse durante un período prolongado.

Los análisis de fiabilidad realizados para la presente investigación confirmaron la estructura de la escala en muestra española: agresividad $\alpha=.97$, hiperactividad $\alpha=.97$ y problemas de atención $\alpha=.92$.

Los estilos educativos parentales se han evaluado mediante el PEF: Escalas de identificación de Prácticas educativas familiares en niños pequeños de Alonso y Román (2003). Este instrumento contiene una escala para personas adultas y otra para niños pequeños de 90 y 15 ítems cada una respectivamente, las cuales recogen la percepción de la estilos educativos de los padres y niños pequeños en el entorno familiar. Las percepciones se recogen a través de cinco situaciones: cuando inician algo nuevo, cuando hay ruptura de rutinas, cuando se van a la cama, cuando cuentan 0 muestran algo y cuando juegan. Estas situaciones interactúan con tres tipos de conflictos: internos, externos y transgresión de normas. Se obtiene un puntaje total para cada estilo parental: autoritario, equilibrado y permisivo. En esta investigación se ha utilizado la escala PEF-H de hijos.

La consistencia interna (fiabilidad) de ambos instrumentos presentan una alfa de Cronbach $(\alpha=.83)$. La validez de contenido $(\alpha=.82)$ y validez de constructo $(\alpha=.83)$ han sido calculadas mediante el grado de acuerdo de expertos (para información estadística más detallada ver Alonso y Román, 2003).

\section{RESULTADOS ALCANZADOS}

Los resultados obtenidos de la realización de una correlación de Pearson entre los estilos parentales evaluados desde la perspectiva infantil y los problemas de conducta se presentan en la Tabla 1.

Tabla 1.

Correlaciones entre los estilos de crianza parentales y los problemas de conducta

\begin{tabular}{lccc} 
& Agresividad & Hiperactividad & Problemas de atención \\
\hline Democrático & -.10 & -.05 & $-.20^{*}$ \\
\hline Permisivo & .12 & .09 & .16 \\
\hline Autoritario & .06 & .04 & $.23^{* *}$ \\
\hline${ }^{*} \mathrm{p}<.05$ (bilateral) ${ }^{* *} \mathrm{p}<.01$ (bilateral) & &
\end{tabular}

El estilo democrático correlaciona significativa y negativamente con los problemas de atención. A pesar de que existe una relación negativa entre el estilo democrático y la agresividad e hiperactividad, esta relación no es significativa.

Por otro lado, el estilo autoritario correlaciona significativa y positivamente con los problemas de atención. No se encontró ninguna relación significativa entre el estilo autoritario y la agresividad e hiperactividad, sin embargo, aunque no significativas, estas relaciones son positivas.

No se encontró ninguna relación significativa entre el estilo permisivo y las diferentes variables (agresividad, hiperactividad y problemas de atención) evaluadas, aunque su relación es positiva.

\section{DISCUSIÓN Y CONCLUSIONES}

Comprobada la relación entre los estilos educativos parentales y los problemas de conducta en edad infantil, coincidiendo con el estudio realizado por Raya, Pino, y Herruzo (2009) y por Flouri, y 
Midouhas (2017), se aboga por la práctica educativa democrática como la más adecuada para el desarrollo óptimo infantil.

Partiendo de la relación significativa y negativa entre el estilo democrático y los problemas de conducta y, partiendo de los beneficios de la práctica deportiva (Pelegrín, et al., 2010), el deporte en familia desde edades tempranas se presenta como factor de protección para canalizar la actividad controlada y ayudar en la regulación de impulsos, contribuyendo a disminuir comportamientos externalizantes de los más frecuentes en la etapa infantil (Campell, 1995). Edmuns, Ntoumanis y Duda (2007) señalan que los sujetos con una alta adherencia a la práctica deportiva revelan una mayor satisfacción de la necesidad psicológica de relacionarse con los demás, pudiendo incidir de manera positiva en la prevención de la ansiedad y depresión, problemas internalizantes más comunes en la etapa preescolar (Kendall et al., 2006).

Aunque hay pocos estudios que relacionen la práctica deportiva en el ámbito familiar como prevención de problemas de conducta en la etapa infantil, son algunas las investigaciones y los proyectos que se están llevando a cabo en esta línea. Así Martinek, y Hellison (2016) realizan una revisión de diferentes estrategias y programas desde el ámbito educativo como el Youth Leader Corps $(Y L C)$ donde se resaltan los beneficios de la práctica deportiva promocionando empoderamiento y responsabilidad en el alumnado participante. Por otro lado el programa Get Ready, llevado a cabo en colegios con índices socioeconómicos bajos trabaja desde la escuela y la familia con el objetivo de trabajar la responsabilidad basada en el aprendizaje a través del deporte (Martinek, y Hellison, 2016). Desde la Universidad de Granada, desde el año 2013, se está llevando un proyecto que reúne a las familias semanalmente en torno a la práctica deportiva. Este proyecto de deporte en familia enfocado a la participación conjunta de madres, padres, niños y niñas, a partir de 3 años, en actividades deportivas, pretende demostrar que la práctica de deporte con los progenitores como modelo tiene una influencia altamente positiva en el desarrollo de los niños y en que sean activos a lo largo de su vida, con las consecuencias a nivel de habilidades prosociales que ello implica (Gutiérrez, 2004). Todavía no hay evidencias científicas acerca de los beneficios de las familias participantes, pero es un proyecto prometedor a nivel nacional.

Abogando por la perspectiva preventiva de intervención planteada por Dishion y Patterson (1992), el programa Aprender a Convivir en Casa (ACC): programa preventivo enfocado a fomentar los factores de protección familiares desde la etapa infantil con el objetivo de prevenir problemas conductuales, delincuencia juvenil y acoso, fomentando la competencia familiar y social (Benavides, Quesada-Conde, López, y Pichardo, 2016); propone el deporte en familia desde la interacción familiar y el tiempo de calidad. Tras la realización de esta investigación, se plantea incluir, de forma más amplia, un apartado dedicado a los beneficios del deporte en familia como estrategia preventiva, vinculada al fomento de conductas respetuosas, disciplinadas, de autocontrol, de liderazgo y autoconfianza, así como a la disminución de problemas de atención y de conductas antisociales, con el objetivo de prevenir los problemas de conducta de los niños y niñas cuyas familias participan en ACC.

Existen varias limitaciones derivadas, principalmente, de la selección de la muestra, pues proviene únicamente de una provincia de España, por lo que los resultados se pueden considerar en cierta medida sesgados. Asimismo, la revisión realizada sobre los beneficios del deporte en familia desde edades tempranas no posee un amplio respaldo debido a las escasas publicaciones actuales.

Como líneas de investigación futuras se propone el estudio de iniciativas y proyectos de manera longitudinal en los que se practique el deporte en familia desde edades tempranas y comprobar su relación con los problemas de conducta. 


\section{AGRADECIMIENTOS}

Investigación realizada gracias a la ayuda para la formación de profesorado investigador (FPI) del Ministerio de Economía y Competitividad, vinculada al Proyecto I+D denominado El programa Aprender a convivir en casa y su influencia en la mejora de la competencia social y la reducción de problemas de conducta (EDU2013-41054-P).

\section{REFERENCIAS}

Abad, M.T., Benito, P.J., Giménez, F.J., y Robles, J. (2013). Fundamentos pedagógicos de la enseñanza comprensiva del deporte: Una revisión de la literatura. Cultura Ciencia y Deporte, 8(23), 137-146.

Achenbach, T. M., y Edelbrock, C. S. (1978). The classification of child psychopathology: A review and analysis of empirical efforts. Psychological Bulletin, 85(6), 1275- 1301.

Alarcón, F., Cárdenas, D., Miranda, M.T., Ureña, M., y Piñar, M.I. (2010). La metodología de enseñanza en los deportes de equipo. Revista de Investigación en Educación, 7, 91-103.

Alonso, J. y Román, J.M. (2003). PEF: escalas de identificación de «prácticas educativas familiares». Madrid: Editorial Cepe.

Balakrishnan, M., Rengasamy, S., y Aman, M.S. (2011). Effect of Teaching Games for Understanding Approach on Students' Cognitive Learning Outcome. International Scholarly and Scientific Research and Innovation, 5(5), 714-716.

Bandura, A. (1989). Social cognitive theory. En R. Vasta (ed.), Annals of Child Development. Greenwich, CT: JAI Press.

Benavides, A., Quesada-Conde, A. B., López, M., y Pichardo, M. C. (2016). Programas de prevención familiar en edades tempranas. Programa aprender a convivir en casa, una propuesta de intervención. International Journal of Developmental and Educational Psychology. Revista INFAD de Psicología., 1(1), 465-474.

Bronfenbrenner, V. (1987) La ecología del desarrollo humano. Barcelona: Paidós. Cicchetti, D., y Lynch, M. (1993). Toward an ecological/transaccional model of community violence and child maltreatment: Consequences for children's development. Psyquiatric, 56, 96-118.

Campbell, S. B. (1995). Behavior problems in preschool children: A review of recent research. Journal of Child Psychology and Psychiatry, 55(1), 113-149.

Cooper, P. (2011). Teacher strategies for effective intervention with students presenting social, emotional and behavioural difficulties: An international review. European Journal of Special Needs Education, 26(1), 7-86.

Dery, M., Toupin, J., Pauze, R., y Verlaan, P. (2004). Frequency of mental health disorders in a sample of elementary school students receiving special educational services for behavioural difficulties. Canadian Journal of Psychiatry, 49(11), 769-75.

Dishion, T. J., y Patterson, G. R. (1992). Age effects in parent training outcome. Behavior Therapy, 23(4), 719-729.

Edmuns, J., Ntoumanis, N. y Duda, J. L. (2007). Adherence and well- being in overweight and obese patients referred to an exercise on pre- scription scheme: A self-determination theory perspective. Psychology of Sport and Exercise, 8, 722-740

Farrington, D. P. (2015). The Developmental Evidence Base: Psychosocial Research. En Crighton, D.A., y J. Towl, G.J. (Eds.), Forensic psychology (pp. 161-181). UK: John Wiley y Sons.

Flouri, E., y Midouhas, E. (2017). Environmental Adversity and Children's Early Trajectories of Problem Behavior: The Role of Harsh Parental Discipline. Journal of family psychology, 31(2), 234-243. 


\section{DEPORTE EN FAMILIA COMO PREVENCIÓN DE PROBLEMAS DE CONDUCTA DESDE EDADES TEMPRANAS}

Galatti, L.R., Reverdito, R.S., Scaglia, A.J., Paes, R.R., y Seoane, A.M. (2014). Sport pedagogy: tension in science and teaching of collective sports games. Revista da Educação Física/UEM, 25(1), 153-162. DOI: 10.4025/reveducfis.v25i1.21088.

González, J., Fernández, S., Pérez, E., y Santamaría, P. (2004). Adaptación española del sistema de evaluación de la conducta en niños y adolescentes: BASC. Madrid: TEA Ediciones.

Gutiérrez, M. (2004). El Valor del deporte en la educación integral del ser Humano. Revista de Educación, 335, 105-126.

Holt, N.L. (2016). Positive youth development through sport. New York: Routledge.

Kendall, P. C., Hedtke, K. A., y Aschenbrand, S. G. (2006). Anxiety disorders. En D.A. Wolfe, y E. J. Mash (Eds.), Behavioral and emotional disorders in adolescents: Nature, assessment, and treatment (pp. 259-299). New York: Guilford Press.

Martinek, T., y Hellison, D. (2016). Learning responsibility through sport and physical activity. En Holt, N.L. (Ed.), Positive youth development through sport (pp. 180-190). New York: Routledge.

Miller, A. (2015). Games Centered Approaches in Teaching Children \& Adolescents: Systematic Review of Associated Student Outcomes. Journal of Teaching in Physical Education, 34, 36-58. DOI: 10.1123/ jtpe.2013-0155.

National Institute for Clinical Excellence. (2014). Antisocial behaviour and conduct disorders in children and young people. London: NICE.

Raya, A. F., Pino, M. J., y Herruzo, J. (2009). La agresividad en la infancia: el estilo de crianza parental como factor relacionado. European Journal of Education and Psychology, 2(3), 211-222.

Reynolds, C. R., y Kamphaus, R. W. (1992). BASC: Behavior assessment system for children: Manual. American Guidance Service, Incorporated.

Rodrigo, M.J., Máiquez, M. L., Martí, J. C., y Byrne, S. (2008). Preservación familiar: un enfoque positivo para la intervención con familias. Madrid: Pirámide.

Rutter, M. (1985). Resilience in the face of adversity: Protective factors and resistance to psychiatric disorder. British Journal of Psychiatry, 147(6), 598-611.

Tur, A., Mestre, V. y Del Barrio, M.V. (2004). Factores moduladores de la conducta agresiva y prosocial, el efecto de los hábitos de crianza. Ansiedad y Estrés, 10, 75-88.

Valera, S., Ureña, N., Ruíz, E., y Alarcón, F. (2010). La enseñanza de los deportes colectivos en Educación Física en la ESO. Revista Internacional de Medicina y Ciencias de la Actividad Física y del Deporte, 40, 1-18. 